\title{
The material-weight illusion revisited
}

\author{
ROBERT R. ELLIS and SUSAN J. LEDERMAN \\ Queen's University, Kingston, Ontario, Canada
}

\begin{abstract}
Experiment 1 documents modality effects on the material-weight illusion for a low-mass object set $(58.5 \mathrm{~g})$. These modality effects indicate that the material-weight illusion is principally a haptically derived phenomenon: Haptically accessed material cues were both sufficient and necessary for fullstrength illusions, whereas visually accessed material cues were only sufficient to generate moderatestrength illusions. In contrast, when a high-mass object set (357 g) was presented under the same modality conditions, no illusions were generated. The mass-dependent characteristic of this illusion is considered to be a consequence of differing grip forces. Experiment 2 demonstrates that the enforcement of a firm grip abolishes the low-mass material-weight illusion. Experiment 3 documents that a firm grip also diminishes perceptual differentiation of actual mass differences. Several possible explanations of the consequences of increasing grip force are considered.
\end{abstract}

Charpentier (1891) first demonstrated that the perceived weight of an object, commonly referred to as its "heaviness," depends not only on its physical mass but also on its size. The larger of two objects of equal mass was consistently reported as lighter. This phenomenon has come to be known as the size-weight illusion. Finding a link between mass and size subsequently prompted other investigators to search for additional factors that might contribute to illusory differences in weight perception.

For example, Dresslar (1894) documented a shapeweight illusion in which objects that were the same in mass, volume, and material but different in shape were judged to be different in weight. Unfortunately, he provided no metric for the variations in shape, rather only vaguely referring to a difference in "compactness."

In 1898, Wolfe documented the material-weight illusion, ${ }^{1}$ which is the subject of the present paper. In this illusion, objects with the same mass but fabricated from different surface materials are judged, on lifting, to weigh different amounts. The general pattern is for same-mass objects of denser materials (i.e., brass) to be judged lighter than same-mass less dense objects (i.e., wood). Ross (1969) explained this effect (together with the size-weight illusion) in terms of expectancies concerning the effects of density on weight perception.

In support, Harshfield and DeHardt (1970) showed that subjects' rankings of expected weight, assessed visually for five different same-size same-mass objects (steel, brass, aluminum, mahogany, and balsa wood), were in the reverse order of rankings of perceived weight after lifting. It should be noted, however, that this was a between-

This work was supported by an NSERC postgraduate fellowship to R. Ellis and by awards from the following institutions to S. J. Lederman: NSERC, The Institute for Robotics and Intelligent Systems Centre of Excellence of Canada, and The Manufacturing Research Corporation of Ontario Centre of Excellence. Correspondence should be addressed to either R. Ellis or S. J. Lederman, The Touch Lab, Psychology Department, Queen's University, Kingston, ON, K7L 3N6 Canada (e-mail: robert.ellis@bangor.ac.uk or lederman@psyc.queensu.ca). groups study in that the subjects who ranked the objects for expected weight did not also rank them for perceived weight via lifting. As such, it could not be determined whether or not perceived weight was in fact based on expectations related to the materials used and/or to direct sensory feedback from the stimulus objects (e.g., density). Harshfield and DeHardt renamed the effect of material on perceived weight as the density-weight illusion, to reflect their emphasis on the role of density in this illusion.

Pick and Pick (1967) and Ellis and Lederman (1993) have shown that the strength of the size-weight illusion varies with the modality that is used to assess the object's size. In short, both studies demonstrated that haptic volume cues were necessary for full-strength illusions. These occurred in either a haptics + vision (traditional paradigm) or a haptics-only (subjects were blindfolded) modality condition. A substantially weaker illusion resulted when an object's volume was presented visually only (visiononly condition). The necessity of haptic volume cues for generating a full-strength illusion suggests that the sizeweight illusion is primarily a haptic phenomenon.

The pattern of modality effects contrasts with that found for the shape-weight illusion: Equally strong shape-weight illusions were generated across all three of the above experimental modality conditions (Ellis, 1996). The primary goal of the present study was to expand our psychophysical investigation of modality effects on perceptual illusions to include the material-weight illusion (Experiment 1). Since the illusion proved to be limited to relatively low-mass objects, we subsequently investigated the role of grip force in the material-weight illusion (Experiment 2) and, more generally, in the perceptual differentiation of weight differences (Experiment 3 ).

\section{EXPERIMENT 1 \\ The Material-Weight Illusion Across Modalities}

Experiment 1 was designed to determine whether material-weight illusions could be generated that were 
Table 1

Description Of Modality Conditions In Experiment 1

\begin{tabular}{|c|c|}
\hline Modality Condition & Description \\
\hline Haptics + Vision & $\begin{array}{l}\text { Direct object grasp with five } \\
\text { fingertips; vision available }\end{array}$ \\
\hline Haptics Only & $\begin{array}{l}\text { Direct object grasp with five } \\
\text { fingertips; no vision }\end{array}$ \\
\hline Vision Only & $\begin{array}{l}\text { Object centered on board, } \\
\text { which was lifted by balancing } \\
\text { it on five fingertips; vision } \\
\text { available }\end{array}$ \\
\hline No Haptics or Vision & $\begin{array}{l}\text { Object centered on board, } \\
\text { which was lifted by balancing } \\
\text { it on five fingertips; no vision }\end{array}$ \\
\hline
\end{tabular}

based solely on haptic or visual cues to object material. The term haptic is used here to denote a broad subsystem of touch, one that uses as inputs feedback from mechanoreceptors embedded in skin, as well as in muscles, tendons, and joints (Loomis \& Lederman, 1986; for a different approach that focuses more specifically on the role of muscles in perception, see Turvey \& Carello, 1995). In the interests of clarity, we emphasize that all perceived weight estimates reported throughout this article were made during lifting. In this sense, they were all haptic weight estimates, as opposed to pure visual weight estimates. The latter form of weight percept has been investigated by Runeson and Frykholm (1981) and by Bingham (1987), who showed that subjects can quite accurately judge the mass of objects by visually observing the kinematic lift profiles of an actor lifting relatively heavy loads. The studies referred to in the general introduction above and all further studies reviewed in this article do not address such visual estimates of weight. Our choice of the phrase vision-only condition indicates that, while the subjects did in fact lift the objects, they were not permitted to directly handle them. Hence, the visual system alone conveyed object properties such as material (as well as size and shape) to the lifter. We further asked whether the same pattern of modality effects initially found in the size-weight illusion would also be present in the material-weight illusion, or whether the pattern of effects would prove to be more similar to those obtained for the shape-weight illusion. Experiment 1 was conceptually and methodologically similar to Experiment 2 of Ellis and Lederman (1993).

Experiment 1 was also designed to determine whether a subject's expectation of weight would be a better predictor of perceived weight than would the actual density of the surface material. According to expectation theory (Ross, 1969), a person might expect that an object made out of a low-density material would weigh less than one of equal mass made out of a high-density material. Therefore, if a lower force were applied to lift a denser object, it would be raised more quickly and easily than the less dense object, leading the observer to judge the former to be lighter than the latter. Empirical support for expectation theory has been mixed at best and, with one excep- tion (Ellis \& Lederman, 1998), has addressed only the size-weight illusion (for a review, see Ellis \& Lederman, 1998). To compare the roles of expected weight versus the physical density of the surface material, all subjects were required to provide estimates of what they would expect the objects to weigh prior to providing estimates of perceived weight after lifting. If these expectations are better predictors than the density of the surface material itself, then the correlation between them and estimates of perceived weight should be considerably stronger than the correlation between density and perceived weight. Also, if there are modality effects in the illusion strengths, they should be mirrored by changes in expectation across modalities. In contrast, however, there should be no difference in illusory magnitude as a function of object mass.

The susceptibility of each modality to the materialweight illusion was assessed by exposing all subjects to four modality conditions (the three experimental conditions above and a control condition), three types of surface material (aluminum, wood, and styrofoam) and two levels of mass ( 58.5 and $357 \mathrm{~g}$ ).

A haptics + vision condition is the traditional paradigm used to assess all illusions of weight. Subjects were allowed to see the object as they directly lifted it to assess its weight. This condition provided baseline data with which effects in the more restrictive modality conditions could be compared.

A haptics-only condition allowed direct hand contact during the lift, but the subjects were blindfolded in order to eliminate visual cues to material differences. Illusions produced in this circumstance would confirm the existence of a purely haptic material-weight illusion. A fullstrength illusion in this condition would indicate that vision is not necessary for a material-weight illusion; furthermore, any diminution of illusion strength between this and the haptics + vision condition would indicate the degree to which vision enhances the illusion.

A vision-only condition restricted surface material cues to the visual system in that no direct contact was allowed between the objects and the subjects' hands. Therefore, illusions generated here would indicate the importance of visual material cues. Furthermore, using a rationale similar to that used above with the size-weight and shapeweight illusions, differences in illusion strength between those produced here and those generated in the haptics + vision condition would demonstrate the extent to which haptic material cues mediate the material-weight illusion.

A control, no-haptics-or-vision, condition combined the restrictive elements of the haptics-only and visiononly conditions. If haptic and visual surface material cues are truly eliminated, no illusion should be reported here. For reference purposes, the four modality conditions are described in Table 1.

\section{Method}

Subjects. Sixteen university students who received course credit for participation in psychology experiments from an introductory psychology course participated in this experiment. All were exper- 
Table 2

Intercorrelation Matrices of $\log _{10}$ Transformed Data (Expected Weight, Perceived Weight, and Surface Density) in the Haptics + Vision, Haptics-Only, and Vision-Only Conditions $(n=16)$ in Experiment 1

\begin{tabular}{|c|c|c|c|c|c|c|c|}
\hline & \multicolumn{3}{|c|}{ Expected Weight } & \multicolumn{3}{|c|}{ Perceived Weight } & \multirow[b]{2}{*}{ Density } \\
\hline & $\mathrm{HV}$ & $\mathrm{H}$ & $\mathrm{V}$ & $\mathrm{HV}$ & $\mathrm{H}$ & $\mathrm{V}$ & \\
\hline \multicolumn{8}{|l|}{$\begin{array}{l}\text { Expected Weight } \\
\text { HV }\end{array}$} \\
\hline $\mathrm{H}$ & $0.953^{\dagger}$ & & & & & & \\
\hline $\mathrm{V}$ & $0.839^{\dagger}$ & $0.875^{\dagger}$ & & & & & \\
\hline \multicolumn{8}{|l|}{ Perceived Weight } \\
\hline HV & $-0.714^{\dagger}$ & $-0.777^{\dagger}$ & $-0.735^{+}$ & & & & \\
\hline $\mathrm{H}$ & $-0.664^{\dagger}$ & $-0.720^{\dagger}$ & $-0.676^{+}$ & $0.833^{\dagger}$ & & & \\
\hline $\mathrm{V}$ & -0.184 & $-0.246^{*}$ & $-0.226^{*}$ & $0.478^{\dagger}$ & $0.557^{\dagger}$ & & \\
\hline Surface Density & $0.788^{\dagger}$ & $0.833^{+}$ & $0.765^{\dagger}$ & $-0.801^{\dagger}$ & -0.802 & $-0.492^{\dagger}$ & \\
\hline
\end{tabular}

Note- $\mathrm{HV}$, scale-equated estimates under the haptics + vision condition; $\mathrm{H}$, scale-equated estimates under the haptics-only condition; $\mathrm{V}$, scale-equated estımates under the vision-only condition. ${ }^{*} p<.05$. $+p<.01$.

imentally naive, all had normal or corrected-to-normal vision, and none reported any muscular or cutaneous problems.

Materials. Six 5.1-cm cubes were used in this experiment. Two were made of aluminum, two of balsa wood, and two of styrofoam. These materials had densities of $2.70,0.14$, and $0.03 \mathrm{~g} / \mathrm{cc}$, respectively. One cube of each of these three materials was produced to have a mass of $58.5 \mathrm{~g}$ (density $=0.44 \mathrm{~g} / \mathrm{cc})$, and the other three had a mass of $357 \mathrm{~g}$ (density $=2.69 \mathrm{~g} / \mathrm{cc}$ ). Note that the former set of density values refers to the density of the surface material of the objects, whereas the latter set of values refers to the overall density of the cubes themselves. This weighting process required some objects to be lightened (hollowed out), others to be weighted, and the heavy aluminum to be kept solid. The walls of the styrofoam and wood cubes were $0.8 \mathrm{~cm}$ thick. The extra mass was added by inserting metal wrapped in cotton batting into the interior of the relevant object. The cotton batting was wrapped around the metal piece to eliminate any movement or rattling of the piece inside the cube. Care was taken to ensure that the mass of all objects was balanced about their centroids and that there were no visual cues that would indicate that the cubes were altered from their solid form.

Procedure. This was a two-part experiment, with three modality conditions in the first part and four modality conditions in the second part. All subjects participated in all conditions of both parts and judged each object once in each condition. They were told to perform all object manipulations with their "dominant hand."

Part 1: Expected weight. In this segment of the experiment the three modality conditions were haptics + vision, haptics only, and vision only. Under each of these conditions, which were presented in random order, the subjects were required to provide an absolute magnitude estimate of what they would expect each of the randomly presented test objects to weigh ${ }^{2}$ (see Zwislocki \& Goodman, 1980). They were told that, for their magnitude estımation, they could assign any number, be it an integer, fraction, or decimal, that they thought best matched the weight of the current object. Neither a standard nor a modulus was used. No time restrictions were imposed on their responses, but an estimate was required before the next object was presented.

Part 2: Perceived weight. In the second part of the experiment, the four modality conditions were haptics + vision, haptics only, vision only, and no haptics or vision. The subjects were asked to lift each of the test objects under each of the modality conditions and to provide an absolute magnitude estimate of weight (see note 2). Both the order of modality and the order of object presentation were determined randomly, although all objects were presented in one modality condition before switching to a new one. In the haptics + vision condition, the subjects were allowed to directly lift the test object and to use vision. In the haptics-only condition, the subjects were allowed to lift the objects but were blindfolded prior to the presentation of the objects. In both of these conditions, the subjects were required to use a five-fingered grasp when liftıng the objects. The arm was not supported in any way, and only a vertical lift was permitted. (In this way, we attempted to minımize rotation about the wrist, although this was not explicitly measured. ${ }^{3}$ ) In the vision-only condition, the subjects were required to balance a thin velour-wrapped plate of wood on their fingertips. The test objects were placed on the center of this plate, and the subjects then hefted the plate and the object. This differed from the vision-only condition used to study both the size-weight illusion (Ellis \& Lederman, 1993) and the shape-weight illusion (Ellis, 1996). In the two previous studies, the objects were suspended from strings, thereby allowing some slip cues. In the no-haptics-or-vision condition, the subjects used the intervening plate as above but were also blindfolded.

\section{Results}

The expected and perceived weight estimates were both scale-equated with the following adjustment. For each subject, each raw score in a given modality condition was divided by the mean of all scores for that subject in that modality condition. This number was then multiplied by the grand mean of all scores (i.e., collapsed across all modality conditions and subjects). These scale-equated values and the density values of the surface materials were converted to base 10 logarithms and used for all subsequent analyses.

The transformed data were then separated into modality conditions and entered into a simple correlation analysis for each of the three experimental groups (haptics + vision, haptics only, and vision only). Table 2 shows that the density of the surface material $(r \mathrm{~s}=-.801,-.802$, and -.492 , respectively; all $p \mathrm{~s}<.01$ ) consistently predicted perceived weight better than did expected weight $(r \mathrm{~s}=$ $-.714,-.720$, and $-.226 ; p \mathrm{~s}<.01, .01$, and .05 , respectively). There was also a strong positive correlation between density and expected weight across all three modality conditions ( $r \mathrm{~s}=.788, .833$, and .765 , respectively). 
Stepwise multiple regression analyses were performed to determine how well these factors predicted perceived weight. The mean regression equation for the haptics + vision condition was:

$\log _{10}$ perceived weight $=-.217 \log _{10}$ surface density

$$
\begin{aligned}
& -.165 \log _{10} \text { expected weight } \\
& +.970,
\end{aligned}
$$

with $R=.812[F(2,45)=43.65, p<.0001]$. Density was a significant predictor of perceived weight $[t(95)=$ $-4.46, p<.0001]$. After its contribution was removed, expected weight was not a significant predictor of perceived weight $[t(95)=-1.54, p=.130]$.

The mean regression equation for the haptics-only condition was:

$$
\begin{aligned}
\log _{10} \text { perceived weight }= & -.250 \log _{10} \text { surface density } \\
& -.147 \log _{10} \text { expected weight } \\
& +.991
\end{aligned}
$$

with $R=.808[F(2,45)=42.27, p<.0001]$. Density was a significant predictor of perceived weight $[t(95)=-4.16$, $p<.0001]$. After its contribution was removed, expected weight was not a significant predictor of perceived weight $[t(95)=-1.07, p=.288]$.

The mean regression equation for the vision-only condition was:

$$
\begin{aligned}
\log _{10} \text { perceived weight }= & -.251 \log _{10} \text { surface density } \\
& +.274 \log _{10} \text { expected weight } \\
& +.395
\end{aligned}
$$

with $R=.548[F(2,45)=9.50, p=.0003]$. Density was a significant predictor of perceived weight $[t(95)=-3.96$, $p=.0002]$. Again, after this contribution was removed, expected weight was not a significant predictor of perceived weight $[t(95)=1.86, p=.069]$.

Because the variable, expected weight, added no significant predictability to perceived weight (after the contribution of density was removed) across the three modality conditions, it was dropped from subsequent analyses. Because expected weight is strongly correlated with the density of surface material, we recognize that the solutions to the multiple regressions above may be unstable. However, we next show that perceived-weight estimates and the magnitude of the material-weight illusion varied substantially both with the mass of the objects and with the sensory modality used. This is in marked contrast to the corresponding expected-weight estimates, which did not vary either with object mass or with sensory modality. We will conclude therefore that it seems most unlikely that expected weight is critical to the material-weight illusion.

Figure 1 shows mean $\log _{10}$ magnitude estimates of weight as a function of the $\log _{10}$ surface density for each modality condition and mass level. The solid lines are linear regression functions fit to the eight data sets.
This figure shows a large discrepancy between the regression lines obtained when the weights of the highmass objects are estimated (the upper four lines) and those obtained when the low-mass objects were estimated (the lower four lines). The regression lines for the high masses, in addition to being higher (indicating that the high-mass objects were judged as heavier than the low-mass objects) also appear to be much flatter. Table 2 confirms that confidence intervals about the means of the slopes for all four modality conditions at the high-mass level include zero (i.e., the slopes are not significantly different from zero). This demonstrates that no reliable illusions were produced at this mass level. Accordingly, no further analysis was performed on this portion of the data.

Conversely, the slopes obtained with the low-mass objects appear to differ across modalities. The slopes are as follows: -0.303 (haptics only), -0.276 (haptics + vision), -0.160 (vision only), and +0.010 (no haptics or vision). Table 3 reveals that, at the low-mass level, only the confidence interval for the control condition (no haptics or vision) contains zero. The confidence intervals for all three experimental conditions, even at $p=.001$, contain only negative numbers, thus indicating a material-weight illusion.

The individual slopes obtained in the four modality conditions at the low-mass level were entered into a oneway within-subjects (modality, four levels) analysis of variance (ANOVA). The results show an omnibus modality effect $[F(3,45)=15.34, p<.0001]$. Three planned nonorthogonal comparisons indicate that the hapticsonly and haptics + vision conditions did not differ from each other $[F(1,15)=2.18, p=.158]$, that the hapticsonly condition produced steeper slopes than the visiononly condition $[F(1,15)=13.39, p=.003]$, and that the slopes obtained under the haptics + vision condition were steeper than those obtained under the vision-only condition $[F(1,15)=7.55, p=.014]$.

An estimate of the illusion strength revealed that when surface density was doubled, magnitude estimates of weight decreased by $21 \%, 23 \%$, and $12 \%$ under the haptics + vision, haptics-only, and vision-only conditions, respectively.

The same statistical procedures used above were followed with the expected-weight data for the low-mass objects in order to ascertain whether or not, at the lowmass level, the reported modality effects for perceived weight would be mirrored by the expected weight data. That is, best-fit linear functions of $\log _{10}$ expected weight estimates as a function of $\log _{10}$ density of surface material were calculated for each subject in each of the three experimental conditions. The resulting slopes were then entered into a one-way within-subjects (modality, three levels) ANOVA. The results indicate that there were no differences in the expected weights across modalities $[F(2,30)=0.63, p=.55]$.

\section{Discussion}

The confidence intervals of the slopes obtained in the four modality conditions at the two mass levels indicate 

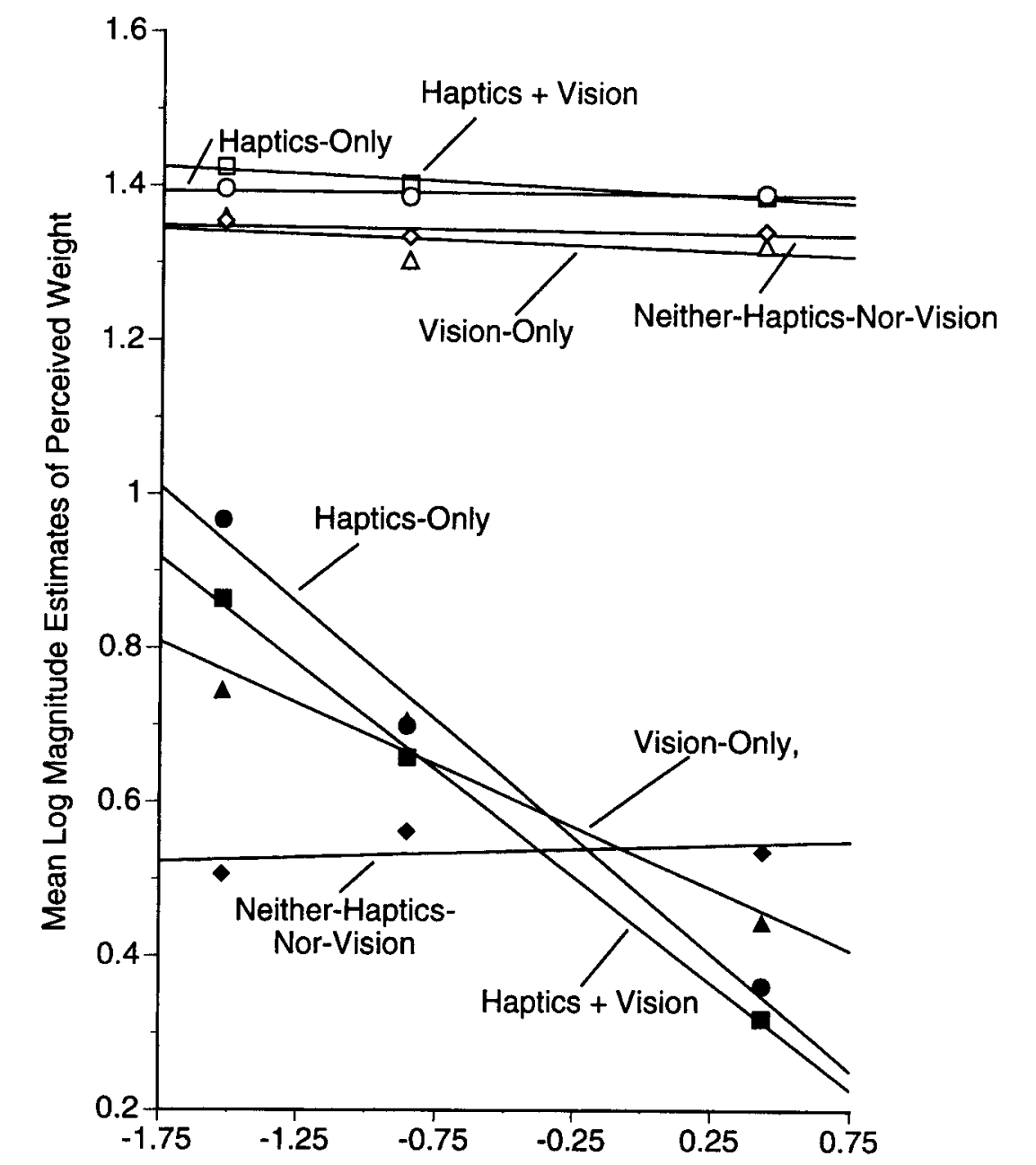

Log Density of Surface Material $(\mathrm{g} / \mathrm{cc})$

\begin{abstract}
Figure 1. Experiment 1: Mean $\log _{10}$ magnitude estimates of weight as a function of the $\log _{10}$ physical density for each modality condition and mass level (58.5 and 357 g). Each datum represents the mean of 16 subjects. The symbols represent the following conditions: D Haptics + Vision, Haptics-Only, $\triangle$ Vision-Only, and $\bullet$ Neither-Haptics-NorVision. The filled and open symbols are used at the low and high mass levels, respectively.
\end{abstract}

that object mass was a determining factor in the production of a material-weight illusion. Illusions occurred only in the three experimental conditions of the low-mass level. As a result, each mass level will be discussed separately.

Low-mass level. The high coefficients of determination for the mean slopes found across the three experimental conditions (shown in Figure 1) demonstrate that density of the surface material was a good overall predictor of perceived weight $\left(r^{2} \mathrm{~s}=.999, .987\right.$, and .938 for the haptics + vision, haptics-only, and vision-only conditions, respectively). The confidence interval for the mean slope obtained in the haptics-only condition serves as an existence proof of a haptic material-weight illusion. This new finding indicates that visual information is not necessary for a material-weight illusion. Further- more, the respective confidence intervals indicate that the haptics + vision, haptics-only, and vision-only modality conditions each produced significant material-weight illusions. All illusions were in the predicted direction: The estimates of weight increased for objects that had decreasing densities of surface material. As expected, the control condition (no haptics or vision) produced no illusion. This indicates that the haptics-only and visiononly conditions adequately eliminated visual and haptic surface density cues, respectively, and that there were no other contaminating cues to surface density (e.g., noise from object manipulation).

The pairwise tests of slopes performed under the three experimental modality conditions indicate the same pattern of illusion strengths reported by Ellis and Lederman 
Table 3

Confidence Intervals About the Means $(n=16)$

by Mass and Modality for the Slopes of the Regressions

of $\log _{10}$ (Estimated Weight) on $\log _{10}$ (Density of Surface Material) in Experiment 1

\begin{tabular}{lll}
\hline \multicolumn{1}{c}{ Modality } & Confidence Interval \\
\hline & High Mass & \\
Haptics + Vision & & -0.038 to $+0.000^{*}$ \\
Haptics Only & -0.018 to $+0.012^{*}$ \\
Vision Only & -0.064 to $+0.034^{*}$ \\
No Haptics or Vision & -0.023 to $+0.011^{*}$ \\
& \\
Haptics + Vision & & -0.443 to $-0.109^{\ddagger}$ \\
Haptics Only & -0.482 to $-0.124^{\ddagger}$ \\
Vision Only & -0.319 to $-0.001^{\ddagger}$ \\
No Haptics or Vision & -0.067 to $+0.087^{*}$ \\
\hline
\end{tabular}

Note-A confidence interval that contains 0 indicates no illusion. ${ }^{*} p<05 . \quad \ddagger p<.001$.

(1993) for the size-weight illusion. The haptics-only condition produced illusions statistically equivalent to those exhibited under the traditional condition of haptics + vision. Moreover, each of these conditions produced stronger illusions than those found in the vision-only condition. As one would expect, given that correlation coefficients are equal to the slopes of standardized data, the weaker vision-only illusion was also more poorly correlated with density than the illusions generated in either the haptics-only condition or the haptics + vision condition ( $r \mathrm{~s}=-.492,-.802$, and -.801 , respectively). We note that this weaker vision-only illusion occurred across both types of weight illusions despite a difference in the visiononly paradigm. Ellis and Lederman (1993) used a stringlift paradigm in the size-weight illusion, whereas this experiment used an intervening plate.

The vision-only condition presented here had two distinct differences from both the haptics-only and the haptics + vision conditions. First, in the vision-only condition, material information could be obtained only by vision; second, no grasp was allowed. This covariation between modality and grasp conditions was accepted for several reasons. First, additional work by Ellis (1996) suggested that grasp information in the vision-only condition was not a significant factor in the strength of material-weight illusions. Specifically, the strength of an illusion generated by a string-lift paradigm, in which the object was lifted by grasping the string to which it was attached, proved to be the same magnitude as that generated by the present intervening-plate paradigm. Second, it was not clear how to devise a technique that used the same direct grasp as that used in the haptic conditions without altering the sensory information in an unacceptable manner. For example, while a transparent cover might provide accurate visual material cues, it could also provide concurrent misleading haptic cues to the object's material. Furthermore, the stimuli would no longer appear homogeneous. Alternatively, one might present the material cues without requiring a grasp in the two haptic conditions by having subjects explore the surfaces either before lifting via the plate or during the lift with the nonlifting hand. Unfortunately, such paradigms force the subject to use haptic modes of touching that are unnatural when assessing the weight of hand-sized objects and thus, are not comparable to the vision-only condition. Third, the present experiment focused primarily on the effects of sensory modality rather than on effects due to the mechanics of grasping. We consider the sensory cues obtained by grasping the object (e.g., size, shape, and texture) to be mediated by the haptic system, and, therefore, their presence in a vision-only condition would compromise our construct of this modality condition.

The findings as a whole demonstrate that, as is the case in the size-weight illusion, it is the haptic cues to surface material that are both sufficient and necessary for a fullstrength material-weight illusion. Visual cues to material are sufficient to generate only moderate illusions.

This pattern of modality effects contrasts sharply with the pattern of modality effects obtained in the shapeweight illusion reported by Ellis (1996). There, statistically equivalent illusion strengths were found under all three modality conditions. In the shape-weight illusion, both haptic and visual cues to shape are sufficient for fullstrength illusions, but neither is necessary.

Although Ellis and Lederman (1998) have shown that subjects' expectations of a difference in mass can influence their subsequent percepts of weight, the effect of modality on the strength of the material-weight illusion cannot be explained in terms of expectancy. The ANOVA on the expected weight data shows that there were no expected weight differences between modality groups that correspond to the significant differences found in the perceived-weight data. The data suggest to us that the mechanism responsible for these modality differences is more likely to be driven directly by sensory information local to the modality being used to derive information about surface material rather than extrapolated from more cognitive global expectations.

High-mass level. It is most intriguing that the confidence intervals for the slopes failed to reveal any material-weight illusions at the high-mass level ( $357 \mathrm{~g})$. This finding too is new. As mentioned earlier, previous researchers have obtained material-weight illusions only by lowering the mass of the denser objects, not by increasing the mass of the less dense objects. This mass dependency contrasts sharply with the size-weight illusion, which has been shown to be quite resilient across a broad range of mass levels.

The effect of mass level on the material-weight illusion provides further support for the importance of sensory influences on this illusion. A strictly cognitive explanation presumably would be impervious to differences in object mass. It would, therefore, have trouble accommodating the observed mass dependency. A sensory-based explanation, on the other hand, assimilates this finding much more easily. It could argue that the material-weight illusion is directly generated by the system that extracts the 
sensory information about object material and massthat is, the haptic system. Experiments 2 and 3 attempted to provide a possible explanation for the absence of a material-weight illusion when the high-mass object set is used.

\section{EXPERIMENT 2}

\section{Grip Force and the Material-Weight Illusion}

A puzzling but intriguing finding of Experiment 1 is that while material-weight illusions were produced for low-mass objects across all three experimental modality conditions, no similar illusions could be demonstrated under these same modality conditions at the high-mass level. What makes the estimates impervious to the illusory effects of surface material at this mass level? One clear-cut difference between mass levels is the grip force required to stabilize the objects while lifting. Because the high-mass objects were six times the mass of the light objects, the minimal grip forces required to stabilize them would have to be six times as great. Perhaps these larger grip forces affect the perception of object weight by saturating the responses of various mechanoreceptor units (e.g., golgi tendon organs in muscles, cutaneous mechanoreceptors, and thermoreceptors), which provide sensory inputs concerning material and mass. Alternatively, a change in stiffness of the limb, which will have increased with the background contraction, may affect weight judgments. While such effects may not hinder the perception of real differences in mass, they may be sufficient to lessen or eliminate illusory differences.

To test this hypothesis, in Experiment 2, subjects judged the weight of the three low-mass objects and three high-mass objects used in Experiment 1, once using a normal grip force and once using a very firm grip force. The normal-grip condition was identical to the haptics + vision condition of Experiment 1. The firm-grip condition required the subjects to use a firm grip while manipulating the objects. This was intended to substantially reduce or eliminate cutaneous, thermal, and/or motor cues to material during the lift; if these cues indeed produce or contribute to the material-weight illusion, then there should be a substantial reduction in the illusion.

In keeping with early tradition, both touch and vision were allowed throughout this experiment. The two grip conditions, which were the focus of the present study, constituted a subset of conditions of an experiment reported by Ellis (1996).

\section{Method}

Subjects. Sixteen university students enrolled in introductory psychology participated as subjects. They received course credit for their participation, and none had participated in Experiment 1. All had normal or corrected-to-normal vision, all were experimentally naive, and none reported or displayed any skin or muscular abnormalities.

Materials. The stimuli were the same as those used in Experiment 1 .
Procedure. All subjects served in both of the grip-manipulation conditions. Under each condition, the six test objects were randomly presented to the subjects, whose task was to assign to each an absolute magnitude estimate of weight (see Experiment 1 for details). The subjects were not provided with feedback of any kind during the experiment. Vision was allowed throughout the experiment.

In all conditions, the subjects were told to use only the dominant hand and were restricted to vertical lifting motions only without the arm being supported in any way. As in Experiment 1, the subjects were instructed not to rotate the objects about their wrists. Under the normal-grip manipulation, the subjects were allowed to directly grip the objects with a light five-fingered grip. In the firm-grip condition, they were again allowed to directly grasp the objects, but they were told to use a firm five-fingered grip. They were further advised to exercise some caution to avoid deforming the objects.

\section{Results}

The perceived-weight estimates were scale-equated as in Experiment 1; these values and the density of the surface materials were converted to base 10 logarithms. The transformed data were used for all subsequent analysis.

Individual regressions of transformed perceived weight on $\log _{10}$ surface density were performed for each subject under both grip manipulations and both mass levels. The resulting slopes were collapsed across subjects and the resulting mean slopes are shown in Figure 2, along with the actual means used to perform the regressions. Inspection of this figure reveals a pattern of slopes somewhat similar to those found in Experiment 1.

The four functions split naturally into two groups of two. The upper two represent the data for the two grip manipulations at the high-mass level. The slopes of these functions are both quite flat. In contrast, considerable diversity is displayed by the lower two slopes, which represent the grip manipulations at the low-mass level. The slope for the normal-grip manipulation is negative $(-0.249)$, whereas the slope for the firm-grip manipulation is very close to zero $(+0.024)$.

In order to ascertain which of these slopes represent genuine illusions, confidence intervals about the means were computed at the high-mass level (see Table 4). These intervals included zero at $p=.05$, and, therefore, no illusions were produced.

At the low-mass level, the confidence intervals for the normal-grip condition excluded zero, even at $p=.001$. This signifies a strong material-weight illusion.

\section{Discussion}

The confidence-interval results provide strong support for the hypothesis that a firm grip abolishes the materialweight illusion. No reliable material-weight illusions were produced in any conditions in which a firm grip was utilized by the subjects (i.e., none of the slopes was greater than zero). The results for the high-mass conditions mirror those obtained in Experiment 1. Support for the hypothesis that the need to use a firm grip when lifting heavy objects abolished the illusion is provided by the results obtained in the firm-grip condition at the low-mass level-no illusion occurred here as well. The high-grip 


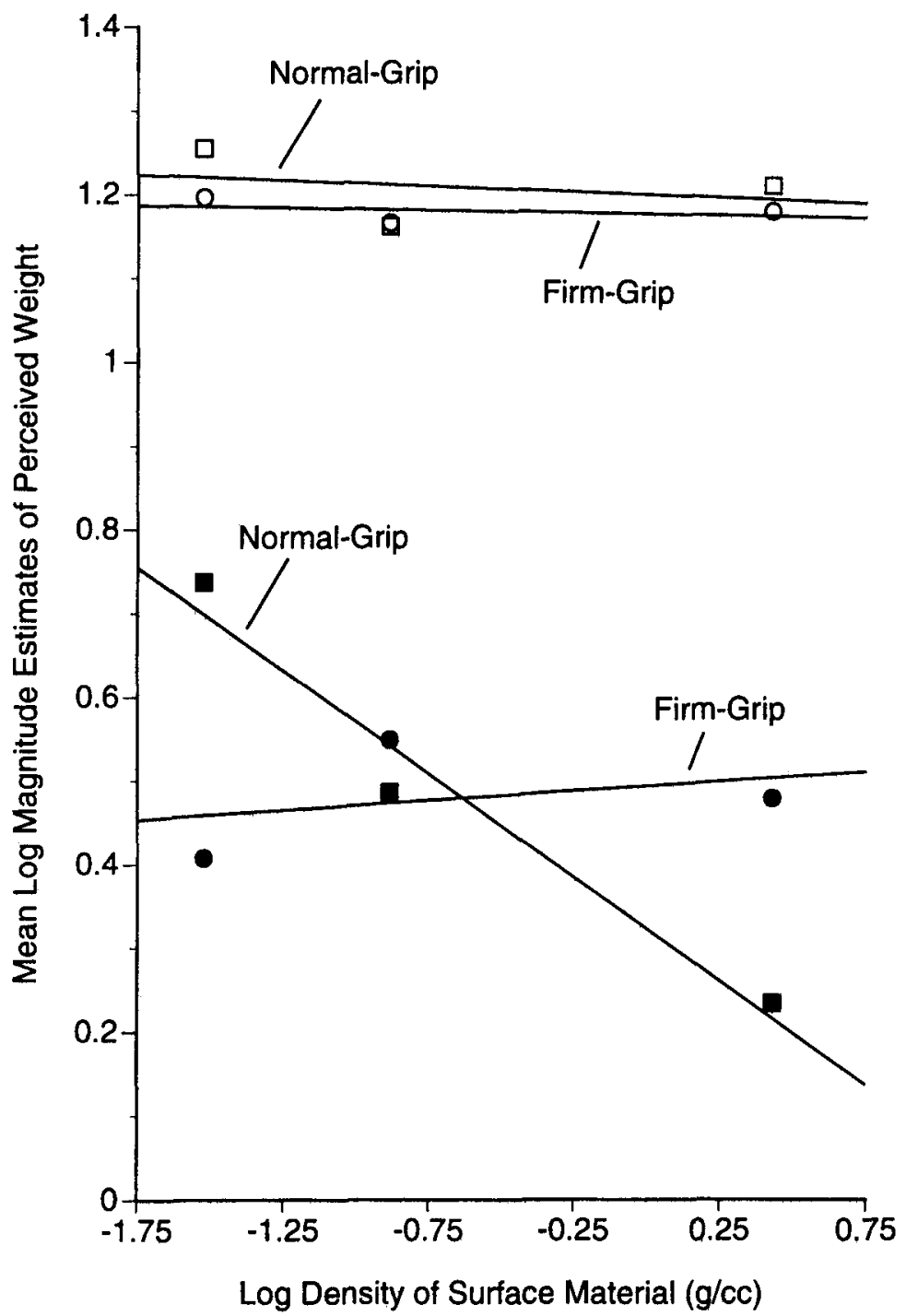

Figure 2. Experiment 2: Mean $\log _{10}$ magnitude estimates of weight as a function of the $\log _{10}$ density of the surface for each grip condition and mass level (58.5 and $357 \mathrm{~g}$ ). Each datum represents the mean of 16 subjects. The symbols represent the following conditions: Normal-Grip and Firm-Grip. The filled and open symbols are used at the low and high mass levels, respectively.

force used may have been sufficient to eliminate or reduce all differences in sensorimotor cues to object material or mass through a process of mechanoreceptor and thermoreceptor saturation and/or increased limb stiffness.

We further note that, in all three of the above conditions, visual material cues were available; apparently, they were not used to generate an illusion. This suggests that the same weight processor processes both haptic and visual inputs about material. Presumably, if there were a separate processor that dealt with visual inputs only, it would not be overloaded and, therefore, should have generated a moderate material-weight illusion.

The normal-grip condition of the low-mass level generated a strong material-weight illusion. Here, although the subjects had both visual and haptic material cues, the modality effects documented in Experiment 1 suggest that the haptic cues to material were used. Information about texture (e.g., Johnson \& Hsiao, 1994) and, possibly, compliance (e.g., Srinivasan \& La Motte, 1994) would likely derive from outputs of slowly adapting Type I units in glabrous skin, whereas thermal conductivity would likely be coded by cutaneous thermoreceptors (e.g., Kenshalo, 1984). However, texture cues may play a somewhat lesser role in differentiating the surface materials. After all, the objects were statically grasped, thereby providing little relative motion between the hand and the object surfaces except for the initial transients. Katz (1925/1989) showed that texture discrimination is best when there is substantial 
Table 4

Confidence Intervals About the Means $(n=16)$ by Mass and Grip Manipulation for the Slopes of the Regressions of $\log _{10}$ (Estimated Weight) on $\log _{10}$ (Density of Surface Material) in Experiment 2

\begin{tabular}{|c|c|}
\hline Grip Manıpulation & Confidence Interval \\
\hline \multicolumn{2}{|c|}{ High Mass } \\
\hline $\begin{array}{l}\text { Normal } \\
\text { Firm }\end{array}$ & $\begin{array}{l}-0.044 \text { to }+0.014^{*} \\
-0.040 \text { to }+0.028^{*}\end{array}$ \\
\hline \multicolumn{2}{|c|}{ Low Mass } \\
\hline $\begin{array}{l}\text { Normal } \\
\text { Firm }\end{array}$ & $\begin{array}{l}-0.334 \text { to }-0.164 \\
-0.074 \text { to }+0.122\end{array}$ \\
\hline
\end{tabular}

Note-A confidence interval that contains 0 indicates no illusion; therefore, statistical significance indicates the presence of an illusion. ${ }^{*} p<.05, \quad \ddagger p<.001$.

relative motion between skin and surface (see also Lederman \& Klatzky, 1987). Presumably, the cutaneous and thermal cues are available when the mass of the object is low enough to avoid any possible effects due to mechanoreceptor saturation and/or increased limb stiffness.

\section{EXPERIMENT 3 Grip Force and Mass Sensitivity}

In Experiment 1, with or without vision, subjects using a normal grip reported a material-weight illusion if the mass of the objects was low $(58.5 \mathrm{~g})$, but not if it was high $(357 \mathrm{~g})$. It was hypothesized that this effect resulted from the subjects' gripping heavier objects with greater force in order to stabilize them during the lift. Such a firm grip may sufficiently overload the responses of various mechanoreceptors to variations in material properties (e.g., texture, hardness, mass) or alternately increase limb stiffness, so that the sensorimotor information normally contributing to the material-weight illusion is diminished. In Experiment 2, when a firm grip was artificially imposed during the lift of the low-mass objects, there was no material-weight illusion. These findings document conditions in which the level of grip force actually distorts judgments of weight.

In Experiment 3, we investigated whether a high-grip force further alters the perception of real differences in mass. If mechanoreceptor and/or thermoreceptor saturation or limb stiffness effects are produced by using a highgrip force, might the ability to resolve real differences in object mass be impaired as well?

To address this final question, subjects were allowed vision to lift a series of graduated masses using both normal and firm grips to provide magnitude estimates of perceived weight. The masses of these objects encompassed the low-mass level of Experiments 1 and 2. A discrepancy in mass differentiation between these two grip-force conditions would establish that grip force plays a more general role in weight perception.

A somewhat nontraditional method, that of magnitude estimation, was used to assess relative perceptual differentiation because of its ease of application and in order to make the methods used in all three experiments comparable. We acknowledge that resolution is usually measured by determining whether or not observers can distinguish a difference between stimuli rather than having them provide a judgment as to how big any difference is. However, when logarithmically transformed and linearly regressed on physical mass, magnitude estimates of weight generally produce high coefficients of determination. Furthermore, the slopes of the resulting regression lines represent the exponent (i.e., the rate of growth of sensation) of S. S. Steven's (1961) power law. We propose to use the exponent as a coarse measure of the system's ability to differentiate along the continuum of mass (for further discussion, see, e.g., Ross, 1997). For any pair of masses, lower slopes signify smaller differences in perceived weight.

\section{Method}

Subjects. Eight university students participated as subjects. All were experimentally naive, all had normal or corrected-to-normal vision, and none reported any muscular or cutaneous problems. None had served as a subject in the previous two experiments.

Materials. The stimuli consisted of eight identical $38.4-\mathrm{g}$ plastic (Tupperware) canisters. Their height was $9 \mathrm{~cm}$, their wall thicknesses were $0.17 \mathrm{~cm}$, and their sides were approximately $6 \times 6 \mathrm{~cm}$. Seven of the canisters were weighted in 10-g increments from 40 to $100 \mathrm{~g}$ and used as test objects. The eighth canister was weighted to $60 \mathrm{~g}$ and was used as a standard. They were weighted with a combination of metal and cotton batting. Care was taken to ensure that this mass was evenly distributed throughout the interior of the canisters and that no shifting or rattling occurred when the canisters were manipulated.

Procedure. All subjects performed the experiment in both conditions (order was counterbalanced) and were allowed both vision and haptics. In the normal-grip condition, the standard canister was placed on a noise-reducing mat in front of the subjects, who were then asked to lift it vertically, without rotating the wrist (as before, the arms were not supported in any way), with a normal five-fingered grip. They were to assign to it a weight value of 100 units. They were then told that they would be given a set of test objects and that they would be required to provide a numerical estimate of weight for each of these objects relative to the standard. A standard was employed so that the estimates of weight could be directly compared with each other without introducing problems pertaining to differences in numerical scale. The subjects could go back and forth between the standard and the test objects as often as they wished, but it was compulsory that they lift the standard prior to each presentation of a new test object. The subjects were presented with two blocks of the seven test canisters, and the order of the canister presentations was randomized withın each block. In the firm-grip condition, the subjects were required to perform the same procedure outlined above but with a firm grip on both the standard and the test objects. They were instructed not to squeeze so tightly that the canisters would deform. At no time during the experiment were the subjects provided with any feedback.

\section{Results}

The mean of the two weight estimates for each object was calculated for each subject under each condition. These means were converted to base 10 logarithms and regressed on the base 10 logarithm of actual weight. A correlated-groups $t$ test was performed on the individual slopes obtained under each condition. The results revealed 


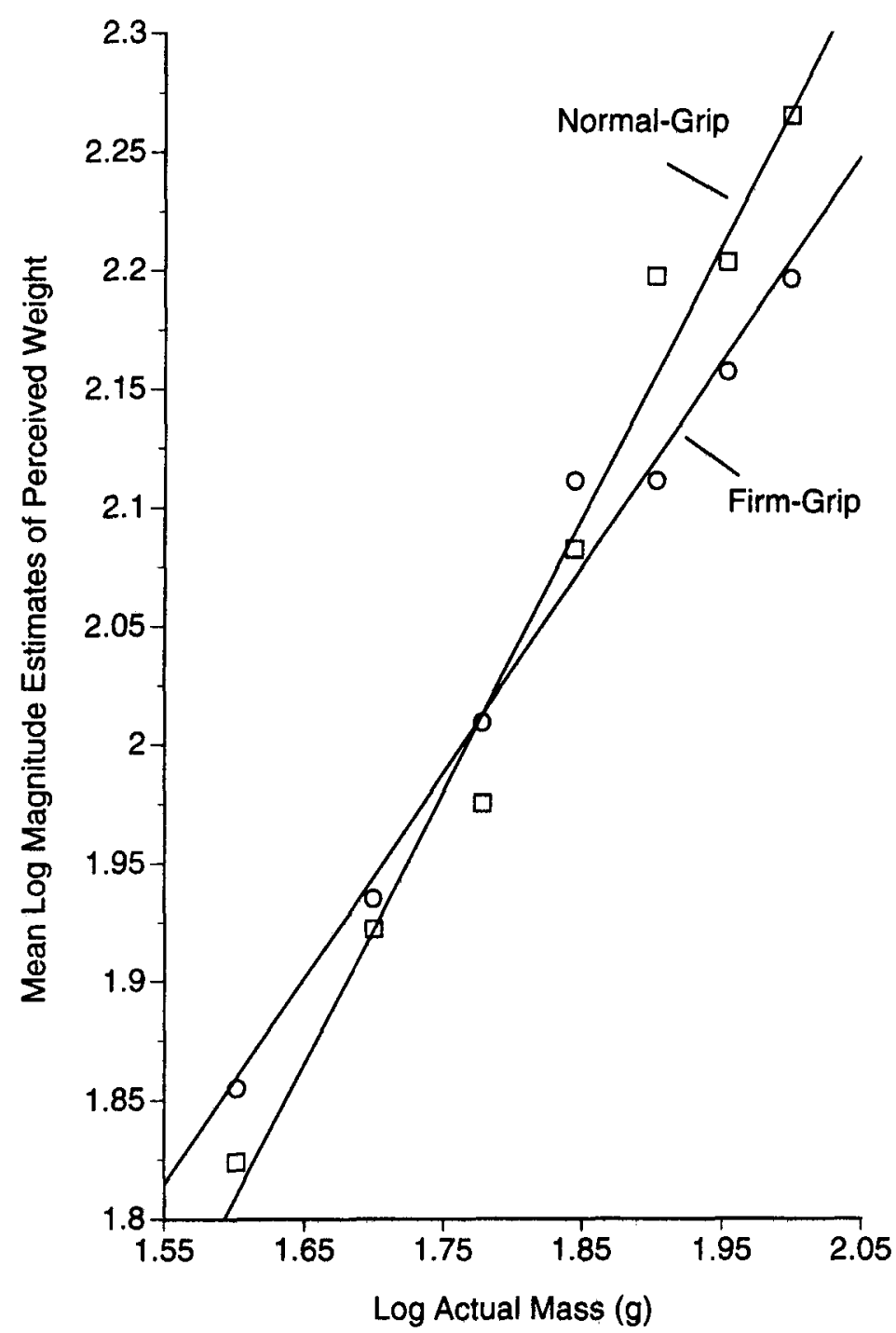

Figure 3. Experiment 3: Mean $\log _{10}$ magnitude estimates of perceived weight as a function of $\log _{10}$ mass under normal- and firm-grip conditions. Each datum represents the mean of 8 subjects. The symbols represent the following conditions: $\square$ Normal-Grip and $\bigcirc$ Firm-Grip.

that a normal grip produced a steeper slope $(M=1.146$, $\left.S D=0.655, r^{2}=.979\right)$ than that obtained with a tight $\operatorname{grip}\left(M=0.865, S D=0.491, r^{2}=.979\right)[t(7)=3.658, p=$ .0081 ]. These mean slopes are shown in Figure 3, along with the means of the actual data on which the slopes are based.

A two-way within-subjects (weight, with seven levels; grip-force, with two levels) ANOVA was performed on the mean $\log _{10}$ estimates of weight for each subject under each condition. The results indicate that the mass of the canister affected its estimated weight $[F(6,42)=21.47$, $p<.0001]$. There was no significant main effect of grip force $[F(1,7)=1.09, p=.3315]$. There was, however, a significant interaction between weight and grip $[F(6,42)=$ $4.85, p=.0010]$. It can be seen that, at the three lower mass levels, the normal-grip condition produced lower estimates of weight than the firm-grip condition, whereas, at the four higher mass levels, the opposite was true. This significant interaction supports the result of the correlated $t$ test of the slopes.

\section{Discussion}

The slope obtained by regressing $\log _{10}$ estimates of weight on $\log _{10}$ actual mass has been shown to vary systematically with volume when volume is held constant across the object set (Cross \& Rotkin, 1975; J. C. Stevens \& Rubin, 1970). The slope increases with increases in volume. The mean slope, representing the exponent of the power function relating apparent weight to actual mass, reported by the subjects under the normal-grip condition 
of this experiment (volume held constant at $324 \mathrm{cc}$ ) is 1.146. This result compares favorably with both the exponent of 1.141 reported by Cross and Rotkin (1975) for objects with volumes of $345 \mathrm{cc}$ and the exponent of 1.143 calculated from the means reported by J. C. Stevens and Rubin (1970) for objects with volumes of $332 \mathrm{cc}$.

The results of both the $t$ test and the ANOVA support the hypothesis that a firm grip also reduces the ability of subjects to perceptually differentiate among physically different masses. A firm grip resulted in a shallower slope than that obtained with a normal grip. This indicates that the subjects judged the objects' masses to be more similar to each other when they were gripped firmly then they did when gripped normally. To the extent that the subjects realized that they were lifting the same objects in both the normal-grip and firm-grip conditions, the observed difference in weight differentiation was likely less than what would be observed if duplicate weight sets that were visually distinct were used. The present effect on perceptual differentiation was not accompanied by a main effect of grip force. However, because the grip force used on the standard matched the grip force used on the objects, had any main effect occurred, it would have been difficult to interpret.

When a high-grip force is used, the reduction in the magnitude of perceived weight differences may account for the lack of a material-weight illusion at the highmass levels of Experiments 1 and 2. When a firm-grip is used (either imposed naturally by the mass of the object or artificially by instructions to the subjects), the mass differentiation by the perceptual system may simply be reduced to the extent that the illusory weight differences due to surface material are masked or undetectable.

A firm grip might affect weight differentiation in a number of different ways. First, it could eliminate slip cues by exceeding the minimum grip force:load force ratio necessary to prevent slip (Westling \& Johansson, 1984). Second, if the grip force is sufficiently high, it could actually saturate activity in the mechanoreceptor (see also Johansson \& Vallbo, 1979; Vallbo \& Johansson, 1984) and thermoreceptor units that normally provide information about variations in material properties (e.g., texture, compliance, thermal conductivity) that potentially cue density. Third, saturation of motor units (e.g., golgi tendon organs) might occur, resulting in changes in mass differentiation. Fourth, mass differentiation might be impaired if subjects also increase arm stiffness when applying a firm grip. Clearly, additional research will be required to assess the role of these factors.

A comparison of the respective slopes reveals that when a firm grip is imposed, subjects' ability to differentiate weights is reduced by $25 \%$. Although it is difficult to make direct comparisons, it is interesting to note that a reduction of this magnitude with a firm grip could well have eliminated or masked any material-weight illusions generated in Experiment 1. There, the reported strengths were $23 \%, 21 \%$, and $12 \%$ for the haptics + vision, hapticsonly, and vision-only conditions, respectively.
The observed association between grip force and weight sensitivity is important to general theories of weight perception as well. Although the effect reported here was generated artificially, in the sense that the subjects were told to grip firmly, the same type of effect can arise in more natural circumstances. Both heavier and smoother objects require higher grip forces to stabilize them while lifting. Since a strong grip force is accompanied by an impairment in differentiating mass, we propose that this perceptual capacity will vary with the grip force employed.

\section{GENERAL DISCUSSION}

\section{Recent Findings Regarding \\ Grip Force and Weight}

Flanagan, Wing, Allison, and Spencely (1995; see also Flanagan \& Wing, 1997) reported an effect of surface texture on weight perception. In short, they found that when subjects lifted a canister covered in satin with a low grip force in a precision grip, they judged its weight to be greater than one covered in sandpaper. Flanagan et al. suggested that the change in perceived weight was due to the fact that subjects employed a higher grip force to grasp the smoother surface, since it was more slippery. The results reported in Experiment 3 of the present study predict that if Flanagan et al. were to have employed their gripforce manipulation across a broad range of weights, they would have found a statistical interaction between object weight and grip force. Note that if a main effect were also found, it would be interpretable only if the interaction was ordinal. Keppel (1982) asserts that a disordinal interaction prevents a general conclusion being reached about the influence of a main effect.

To establish whether a main effect of grip force (as specified by variations in slip) on weight could fully explain the material-weight illusion reported in Experiments 1 and 2 of the present study, the following miniexperiment was performed. Strips of the materials used in Experiments 1 and 2 were tested on 6 subjects in order to measure the relative slipperiness between these materials and the fingertips. This was done by measuring the normal force required to cause these surfaces to begin to shear across two fingertips (index and middle) when a $60-\mathrm{g}$ mass was applied to the fingertips (for a detailed description of the force control, balance apparatus, and the technique used to determine relative shear forces at the point of slip, see Lederman, 1978). Each stimulus was placed on spherical glass beads, which lined the surface of a glass plate, that was placed on one end of the balance arm. Counterweights were adjusted at the other end so that the subjects had to apply a normal force of $0.589 \mathrm{~N}$ on the stimuli to keep the balance arm level. As the subject did so, weights were added to a small container, which was suspended on a thread over the side of the stimulus to increase the horizontal force acting tangentially between the fingertip skin and the stimulus. Because the friction force between the base of the stimulus and the glass beads was very nearly zero, the normal force required to just 
move the stimulus across the fingertips represented the magnitude of shearing force required to just overcome friction between that material and the fingertips. The results of these tests revealed that the mean force required to initiate slip between aluminum, foam, and wood and the fingertips was $0.861 \mathrm{~N}(S D=0.031), 0.895 \mathrm{~N}(S D=$ $4.046)$, and $0.768 \mathrm{~N}(S D=0.023)$, respectively. A oneway within-subjects (material, three levels) ANOVA of the shear-force data revealed an overall effect of material on shear force $[F(2,10)=23.91, p=.0003]$. Orthogonal comparisons further revealed that the aluminum surface was not reliably different in slipperiness from the styrofoam surface $[F(1,5)=2.54, p=.171]$ and that the wood surface was more slippery than the combination of aluminum and foam $[F(1,5)=60.1895, p=.001]$.

These results indicate that the grip-force effect reported by Flanagan et al. (1995) and Flanagan and Wing (1997) does not underlie the material-weight illusion reported here. Their findings, coupled with the shear-force data reported immediately above, would predict that the wood cube (the most slippery surface) should be judged to be heavier than both the aluminum and the styrofoam cubes, which should be judged to be the same. In contrast, in the present study, the styrofoam cube was judged to be heaviest, followed by the wood cube, and finally the aluminum cube.

Flanagan et al. (1995) further employed a control condition (horizontal grip) to ensure that the effect they obtained was a result of differences in slip per se and not a result of texture or other material cues (e.g., density). The horizontal-grip condition required subjects to place the thumb underneath the object and the index finger on top. This selectively eliminated slip cues while retaining all other cues to surface material. In this condition, there was no effect of texture on perceived weight, confirming a role for slip but not material in their experiments. Thus, it seems the effect they reported and the effect reported in this study are orthogonal.

We do in fact note a potentially significant difference between the two studies in terms of the stimuli employed. Flanagan et al. (1995) used canisters wrapped with two different materials, satin or sandpaper. We speculate that subjects would be less likely to perceive such objects to be made of a single homogeneous material. The same speculation may apply as well to the more recent study by Flanagan and Wing (1997), who used thin chips that were also covered in either satin or sandpaper and attached to a three-dimensional force transducer. Presumably, it would be highly salient to the subjects that they were lifting a fabricated, nonhomogeneous "object." Conversely, in the present study, the stimuli appeared (both haptically and visually) to be homogeneous unaltered objects. It could be that this distinction is critical to the processes used to determine perceived weight in the two studies. Material-weight illusion mechanisms may be activated only if the material cues are considered to uniformly represent a gestalt object rather than merely a local feature attached to the "object." The stimuli used by
Flanagan and his colleagues (Flanagan \& Wing, 1997; Flanagan et al., 1995) may limit the influence of surface density on weight perception and allow for the emergence of a more subtle local property, such as slipperiness (which does influence grip force), to become influential. J. R. Flanagan (personal communication, October 30, 1996) concurs that the difference between the processing of a whole "object" as opposed to the processing of a feature (slipperiness) that is local to a small portion of that object may be a critical component of resulting percepts of weight.

\section{Perception and Action}

Much research has been performed on hand-object interactions, although primarily from either a purely sensory/perceptual perspective or a purely motor perspective. However, an understanding of hand function must additionally focus on both sensory/perceptual influences on manual control (perception for action) and, conversely, on motor contributions to manual sensing and perceiving (action for perception; see Lederman \& Klatzky, 1997). There has been relatively little attention paid to the influence of manual exploration on perception until somewhat recently. In their work on haptic perception and recognition, Lederman and Klatzky (e.g., Lederman \& Klatzky, 1987) have emphasized the importance of systematic hand-movement patterns (exploratory procedures) in constraining the type and precision of sensory information available about objects and surfaces. For example, moving the hand repetitively back and forth across the interior surface of an object (a "lateral motion" exploratory procedure) provides precise information about surface texture but little, if any, information about object weight. To learn about an object's weight, one needs rather to lift the object away from any supporting surface and possibly to heft it up and down (an "unsupported holding" exploratory procedure). Additional support for the importance of manual action on haptic perception is provided by the present study: Here, we have shown that changing the way in which a person grasps an object (e.g., firm vs. light grasp, and side vs. top-bottom fingertip grasp, as shown by Flanagan et al., 1995) may alter the perceived weight of an object.

\section{Summary}

The present study demonstrates that the materialweight illusion is mass dependent. Strong illusions were generated at a low-mass level, whereas no illusions were generated at a high-mass level. Furthermore, at the lowmass level, the illusion was principally driven by haptic (cutaneous) information. Vision-only material-weight illusions were significantly weaker than either haptics + vision or haptics-only illusions. High-grip force was shown both to eliminate the low-mass illusion and to reduce perceptual differentiation of actual differences in mass. The effects of grip force on weight perception demonstrated in Experiments 1-3 cannot be fully explained by the elimination of slip cues. We have speculated 
on a number of potential factors, whose contributions will be considered in future work on this topic.

\section{REFERENCES}

BingHAM, G. P. (1987). Kinematic form and scaling: Further investigations on the visual perception of lifted weights. Journal of Experimental Psychology: Human Perception \& Performance, 13, 155-177.

ChARPENTIER, A. (1891). Analyse experimentale de quelques elements de la sensation de poids [Experimental study of some aspects of weight perception]. Archives de Physiologie Normales et Pathologiques, 1.3, $122-135$

Cross, D. V., \& Rotkin, L. (1975). The relation between size and apparent heaviness. Perception \& Psychophysics, 18, 79-87.

Dresslar, F. B. (1894). Studies in the psychology of touch. American Journal of Psychology, 6, 313-368.

ELLIS, R. R. (1996). Haptic weight illusions with and without vision. Unpublished doctoral dissertation, Queen's University, Kingston, Ontario, Canada.

ELLIS, R. R., \& LEDERMAN, S. J. (1993). The role of haptic versus visual volume cues in the size-weight illusion. Perception \& Psychophysics, 53, 315-324.

Ellis, R. R., \& Lederman, S. J. (1998). The golf-ball illusion: Evidence for top-down processing in weight perception. Perception, 27, 193-201.

Flanagan, J. R., \& WING, A. M. (1997). Effects of surface texture and grip force on the discrimination of hand-held loads. Perception \& Psychophysics, 59, 111-118.

Fi.anagan, J. R., Wing, A. M., Allison, S., \& Spencely, A. (1995). Effects of surface texture on weight perception when lifting objects with a precision grip. Perception \& Psychophysics, 57, 282-290.

HarshField, S. P., \& DeHardT, D. C. (1970). Weight judgment as a function of apparent density of objects. Psychonomic Science, 20, 365-366.

Johansson, R. S., \& VAllBo, A. B. (1979). Tactile sensibility in the human hand: Relative and absolute densities of four types of mechanoreceptive units in glabrous skin. Journal of Physiology, 286, 283300.

JoHNSON, K. O., \& HsiaO, S. S. (1994). Evaluation of the relative roles of slowly and rapidly adapting afferent fibers in roughness perception. Canadian Journal of Physiology \& Pharmacology, 72, 488-497.

KATZ, D. (1989). The world of touch (L. E. Krueger, Trans.). Hillsdale, NJ: Erlbaum. (Original work published 1925)

Kenshalo, D. R. (1984). Cutaneous temperature sensitivity. In W. W. Dawson \& J. M. Enoch (Eds.), Foundations of sensory science (pp. 419-464). Berlin: Springer-Verlag.

KEPPEL, G. (1982). Design and analysis. A researcher's handbook (2nd ed.). Englewood Cliffs, NJ: Prentice-Hall.

Lederman, S. J. (1978). "Improving one's touch" ... and more. Perception \& Psychophysics, 24, 154-160.

LEDERMAN, S. J., \& KLATZKY, R. L. (1987). Hand movements: A window into haptic object recognition. Cognitive Psychology, 19, 343-368.

Lederman, S. J., \& KlatzKY, R. L. (1997). Haptic aspects of motor control. In F. Boller \& J. Graftman (Eds.), Handbook of neuropsychology (Vol. 11, pp. 131-148). Amsterdam: Elsevier.

LoOmis, J. M., \& Lederman, S. J. (1986). Tactual perception. In K. Boff, L. Kaufman, \& J. Thomas (Eds.), Handbook of perception and human performance (chap. 31, pp. 31-1-31-41). New York: Wiley.

PICK, H. L., \& Pick, A. D. (1967). A developmental and analytic study of the size-weight illusion. Journal of Experimental Child Psychology, 5, 362-371.

Ross, H. E. (1969). When is weight not an illusion? Quarterly Journal of Experimental Psychology, 21, 346-355.
Ross, H. E. (1997). On the possible relations between discriminability and apparent magnitude. British Journal of Mathematical \& Statistical Psychology, 50, 187-203.

Runeson, S., \& Frykholm, G. (1981). Visual Perception of Lifted Weight. Journal of Experimental Psychology: Human Perception \& Performance, 7, 733-740.

Srinivasan, M. A., \& LaMotte, R. H. (1994). Tactual discrimination of softness. Journal of Neuroscience, 72, 88-100.

Stevens, J. C., \& Rubin, L. L. (1970). Psychophysical scales of apparent heaviness and the size-weight illusion. Perception \& Psychophysics, 8, 225-230.

STEVENS, S. S. (1961). To honour Fechner and repeal his law. Science, $133,80-86$.

Turvey, M. T., \& Carello, C. (1995). Dynamic touch. In W. Epstein \& S. Rogers (Eds.), Handbook of perception and cognition (Vol 5, pp. 401-490). New York Academic Press

Vallbo, A. B., \& Johansson, R. S. (1984). Properties of cutaneous mechanoreceptors in the human hand related to touch sensation Human Neurobiology, 3, 3-14.

Westling, G., \& Johansson, R. S. (1984). Factors influencing the force control during precision grip. Experimental Brain Research, $53,277-284$

Wolfe, H. K. (1898). Some effects of size on judgments of weight. Psychological Review, 5, 25-54.

Zwislocki, J. L., \& Goodman, D. A. (1980). Absolute scaling magnitudes: A validation. Perception \& Psychophysics, 28, 28-38.

\section{NOTES}

1. The material-weight illusion has been traditionally explained in terms of density differences, with little, if any, mention of other material properties. Such an emphasis on density is not surprising, given that it is determined solely by mass and volume, both of which influence the visual and/or haptic perception of weight (e.g., Ellis \& Lederman, 1993; Pick \& Pick, 1967). Yet changes in material (i.e., wood to brass) are accompanied by variation in several additional properties, as well as density (e.g., thermal conductivity, compliance, texture, and color). Strictly speaking, therefore, these covariations may be thought of as confounds in a density-based explanation of the illusion. However, it may be neither possible nor even productive to eliminate them, since such property covariation typically occurs naturally. Accordingly, we prefer to retain the more inclusive term material-weight illusion in the present paper, while reporting effects of material variation on perceived weight in terms of quantitative variation in surface density.

In early research on the material-weight illusion, weight was incorrectly reported in units of mass rather than force. In this paper, we use the term mass to describe the physical property; however, to link with the results of earlier research, we use the term weight in instructions to subjects to refer to their perceptual judgments of mass. As well, this would seem to make sense because, in general, most people are less familiar with the term mass, and do not properly understand its scientific meaning.

2. The present study reports perceptual judgments of perceived weight that were made in response to instructions to estimate "weight." Whether the subjects judged weight or some other direct correlate(s), such as lift force or hand velocity, cannot be resolved in the present study.

3. The instruction not to rotate the wrist may not have eliminated the possible influence of rotational inertia.

(Manuscript received November 15, 1996, revision accepted for publication September 12,1998.) 